\title{
Visualization of the healing process on reinforced concrete beams by application of Digital Image Correlation (DIC)
}

\author{
E. Tsangouri ${ }^{1}$, K. Van Tittelboom ${ }^{2}$, D.Van Hemelrijck ${ }^{1}$ \\ $\&$ N. De Belie ${ }^{2}$ \\ ${ }^{1}$ Department of Mechanics of Materials of Constructions (MeMC), \\ Vrije Universiteit Brussel (VUB), Belgium \\ ${ }^{2}$ Magnel Laboratory for Concrete Research, Ghent University, Belgium
}

\begin{abstract}
Fabrication of concrete with self-healing capabilities has recently become a hot research topic. In general, material science is focused on the development of smart engineering concrete and cementitious composites with an extended service life. Indeed, materials that remain durable and keep their mechanical performance, damage mechanisms occurring should heal by themselves. In the case of this study, formation of damage and recovery of the mechanical properties is investigated by application of an encapsulated healing agent. On an experimental level, it is imperative to implement an optical, non-contact and online technique to visualize and compare the crack propagation at the loading and reloading (when the initial cracks are filled by the healing agent) stage. For that reason, optical measurements by application of Digital Image Correlation (DIC) are performed during the tests. Processing images captured by a 4-digital cameras system during all the loading stages of four-point bending tests give a full- field view of the crack displacement and strain profiles. A step further, the visualization of the cracking phenomena by DIC offers a useful tool to apply fracture theories of concrete on healing systems.

Keywords: reinforced concrete, self-healing, encapsulation, cracking, DIC.
\end{abstract}




\section{Introduction}

The need for innovative building materials that resist to damage, time, weather and loading conditions and extend their service life is imperative. This demand has led several laboratories worldwide, following the philosophy of smart selfhealing ( $\mathrm{SH})$ systems, to focus their research topic on advanced materials that can recover their mechanical properties after damage. More specific, in concrete technology, the recent 20 years, investigation is done in autogenous and autonomous healing concepts. In both cases, the quantitative characterization of the healing process is a hot area of research.

Experimentally, a method is required that gives a full-field view of the crack formation (main cause of damage), visualizes the mechanical behavior and highlights the recovery of material properties due to the healing procedure. In the case of this study, the healing phenomena appeared on an already successfully applied SH system is investigated in practice by application of Digital Image Correlation (DIC), an already well-established and promising Non Destructive Testing (NDT) optical technique.

\section{Self-healing system}

In recent years, the research team of the Magnel Laboratory at the University of Ghent has been working on the encapsulation of a polyurethane-based 2component healing agent into cementitious materials $[1,2]$. Till now, hollow glass or ceramics tubes filled with healing agent were embedded into cementitious mortar beams of small dimensions. The healing efficiency was measured with different methods ( $\mu$-CT, X-Ray, Acoustic Emission) and the optimized SH system set-up was defined. A step further in this study, this encapsulated system was applied on reinforced concrete beams and the success of it on real-scale tests was investigated. According to the main idea of the aforementioned smart material systems, the formation of cracks, during fourpoint bending in our case, is breaking the embedded capsules, releasing the healing agent. The open cracking area is filled with the agent and the defected area reverts after a certain curing time. Later on, reloading of the healed element gave a clear view of the regain in mechanical properties $[1,2]$.

\section{Digital Image Correlation}

DIC was developed in the 1980s and since then has been well improved and established. The basic principles and theoretical background of this on-line and full-field view optical method are well-known and described in detail in literature [3]. The DIC system consists of 2 cameras (-CCD) and a data acquisition system and requires a random speckle pattern attached to the material. A number of images are captured by cameras and via a digitizing board, the analog signal is converted into an integer gray level intensity value for each pixel of the image. The light intensity is measured on a range between 0 and 255 (black to white). The grey scale images of an object during loading are 
compared by an image correlation piece of software which calculates the displacement of regions in each image relatively to the reference image. Then, the strains are derived from the displacement gradients [3]. DIC capturing options appear promising in the case of this study, since crack opening can be measured as the deformation of certain points of the image at several loading stages and the strain profiles of loading and reloading stage indicate the damaged area and the potential recovery of it after healing.

\section{Testing material}

Rectangular-shaped concrete beams with dimensions of $650 \times 100 \times 100 \mathrm{~mm}^{3}$ were manufactured. Steel bars applied as bending reinforcement (at a level of $100 \mathrm{~mm}$ above the bottom of the beam) and short steel fibers randomly distributed at the whole beam prevent extension of shear cracks that may appear during loading. The geometry of the beam and the reinforcement were chosen to create cracks, during loading, wide enough to break the capsules and at the same time to restrict the crack opening in less than $1.00 \mathrm{~mm}$ so that the areas will be easily filled with the limited amount of agent carried by capsules. Short and longer glass or ceramic hollow tubes (capsules), carrying the healing agent, were placed at the steel bar reinforcement level randomly distributed at the whole length of the beam. Different series of beams were manufactured depending on the capsules material (glass/ceramic), the length of the tubes, and the cover of capsules (mortar layer, cement paste bar or nothing). A series of beams, made without any encapsulation system were loaded and reloaded and are used for reference. The material characteristics are summarized in the following Table 1.

Table 1: $\quad$ Material characteristics.

\begin{tabular}{|ll|}
\hline Concrete & \\
\hline $\begin{array}{l}\text { CEM I, 52.5 N, w/c }=0.5 \\
\text { Reinforcement }\end{array}$ & Compressive strength $=58.6 \mathrm{~N} / \mathrm{mm}^{2}$ \\
\hline 2 steel bars & $\mathrm{d}=6 \mathrm{~mm}$ at height $=10 \mathrm{~mm}$ from the bottom \\
Steel fibers & Randomly distributed, $\mathrm{d}=0.71 \mathrm{~mm}$ \\
Healing system & \\
\hline Glass/ceramic tubes & Short $(60 \mathrm{~mm}) /$ Long $(400 \mathrm{~mm})$ \\
Healing agent & Polyurethane-based 2 component \\
\hline
\end{tabular}

\section{Experimental set- up}

\subsection{Four-point bending test set-up}

All the beams were tested under four-point bending (upper loading span of $200 \mathrm{~mm}$ ). The displacement-controlled loading was stopped when $4.00 \mathrm{~mm}$ of 
deflection was reached. The beams were cured in room temperature for 24 hours and then reloaded following the same procedure. Apart from DIC on-line monitoring, Acoustic emission measurements were done. An 8-sensors system was attached at the surfaces of each beam and then a 3D location analysis was done to locate the capsules breakage and characterize on en energy level the crack formation of the virgin and the healed beams. Furthermore, after testing, permeability tests were performed to compare the water penetrating into the initial and healed cracks. Analysis of the results obtained from the aforementioned techniques, combined with the load-displacement curves derived out of the INSTRON testing machine give an integrated overview of the healing procedure. Due to the limited size of this publication, acoustic emission and permeability test results are not presented in this paper.

Finalizing the experimental investigation, the series of beams tested are categorized into three different groups according to the DIC analysis giving a representative overview of the healing efficiency.

\subsection{Digital Image Correlation set-up}

Two different DIC systems, containing 2-CCD cameras each, are used to visualize the crack formation. One of the DIC cameras systems was monitoring deformation phenomena at the bottom of the beams. The other, was facing the side of the beam giving an overview of the cracks formatting between the two upper loading supports (Fig. 1). The technical characteristics and the set-up of the cameras are shown in the following table (Table 2) and the figure respectively. Almost 1000 images were captured during each loading test. Deformation analysis was conducted using the software package VIC3D-2009, which compared the speckle pattern of the deformed beam obtained during loading or unloading with a reference pattern. During post-processing analysis, the speckle pattern area under investigation is defined as Area of Interest (AOI). Every pixel of this area stores a certain grey scale value, corresponding to the intensity of the light reflected by the surface. The varied grey value pattern is obtained on different square groups of pixels (subsets). The image correlation software locates every subset of the initial image in the deformed image. In this way, deformation measurements were transformed into digital correlation calculation and the displacement vector of each subset was obtained by subtracting the new coordinates from the original ones.

During the bending test, displacement fields (U, V, W (mm)) at three directions $(\mathrm{X}, \mathrm{Y}, \mathrm{Z})$, the coordinates of each point of the area of interest (AOI) measured in $\mathrm{mm}$ and/or pixels, the sigma error of the correlation and the load of the INSTRON machine were derived [3]. Furthermore, requesting for strain profiles, the displacement fields are differentiated to calculate the normal and shear strains (exx, eyy, exy (\%, unity, micro strain)) and the principal strains (e1, e2 based on different criteria).In the case of this study, only deformation (X) and strain (exx -unity) results out of the DIC-system at the side of the beam are presented. 

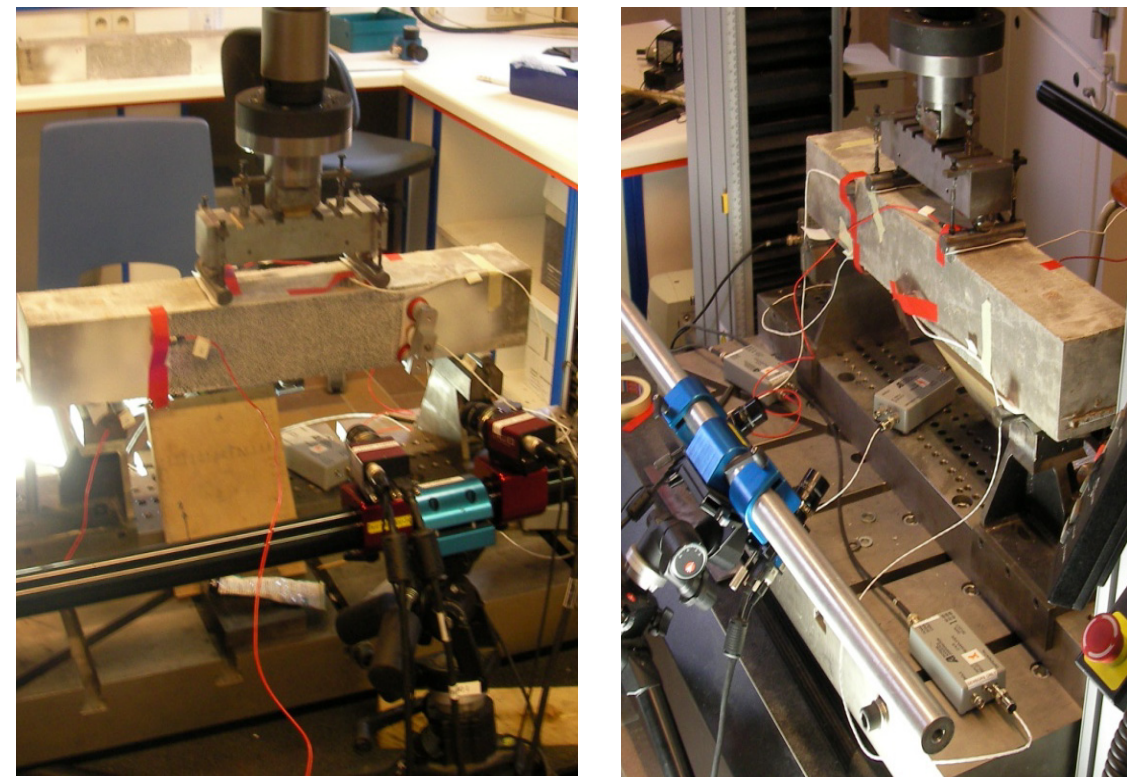

Figure 1: Digital Image Correlation 2-cameras system set-up.

Table 2: $\quad$ Digital Image Correlation technical characteristics.

\begin{tabular}{|ll}
\hline CCD camera type: & AVT Stingray \\
Lenses: & $23 \mathrm{~mm}$ \\
Resolution: & 2456 x 2058 \\
Focused area: & bottom system: $130-150 \mathrm{~mm}$ \\
& side system: $250 \mathrm{~mm}$ \\
Time capture: & every 2 seconds \\
\hline
\end{tabular}

\section{Experimental results}

Taking into account the load- displacement curves measured by INSTRON machine and the strain and crack opening profiles of DIC, beams that exhibited healing can be categorized into the following groups:

- Healed beam - case 1: not reopening of the initial crack and a new crack is formatted during reloading

- Healed beam - case2: crack reopening during reloading but less wider than at the loading stage 
These two healing phenomena are compared with the reference beam behavior. Since the choice of the appropriate SH encapsulated system is not the main purpose of this study, representative results from one beam each time are used to explain in practice the healing process for both aforementioned categories.

In the following graphs (Figs. 2-4), the load-displacement curves as captured by the INSTRON machine are presented for the reference beam and the two cases of healed beams.

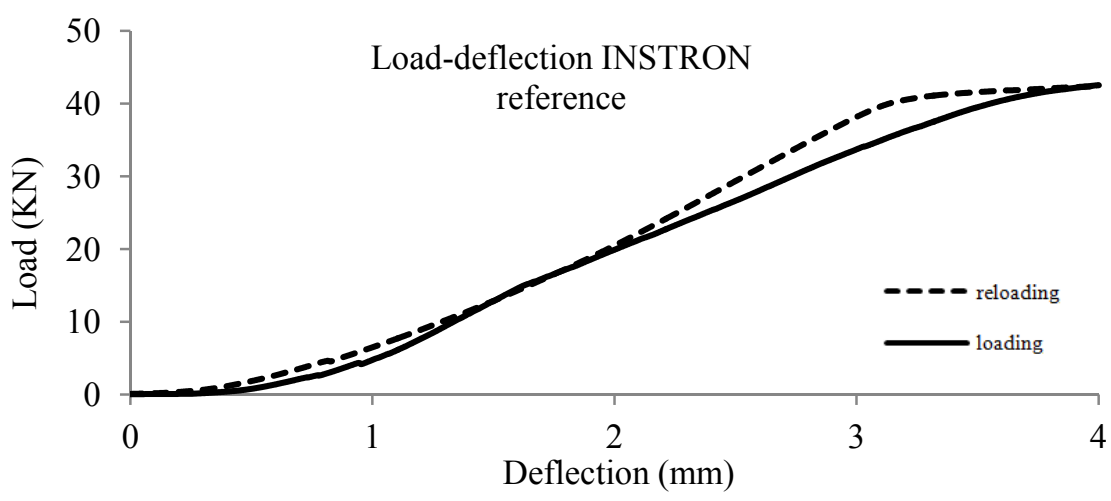

Figure 2: Load-deflection curve of the reference beam.

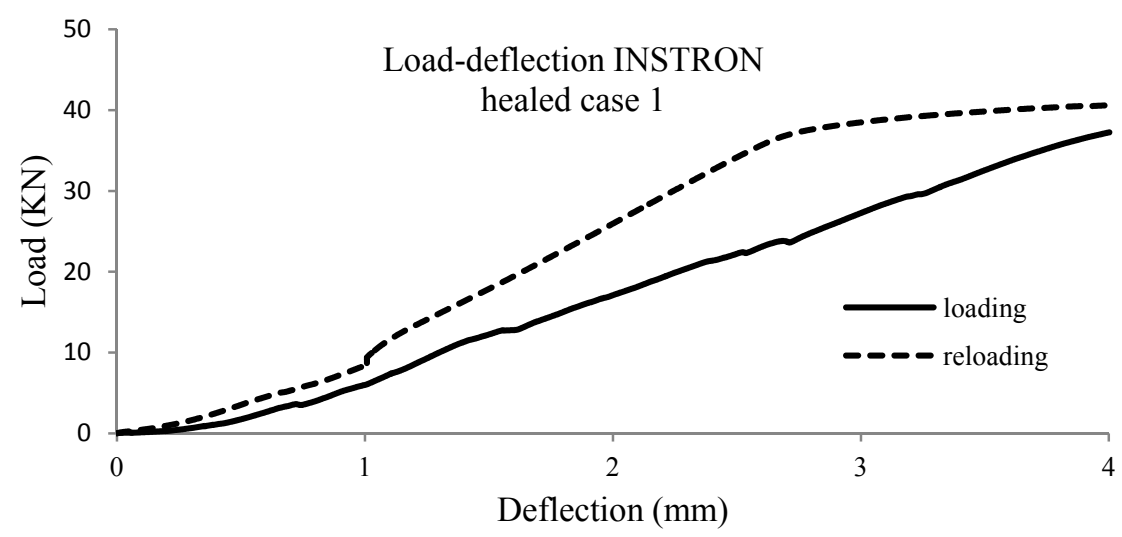

Figure 3: Load-deflection curve of the healed beam - case 1. 


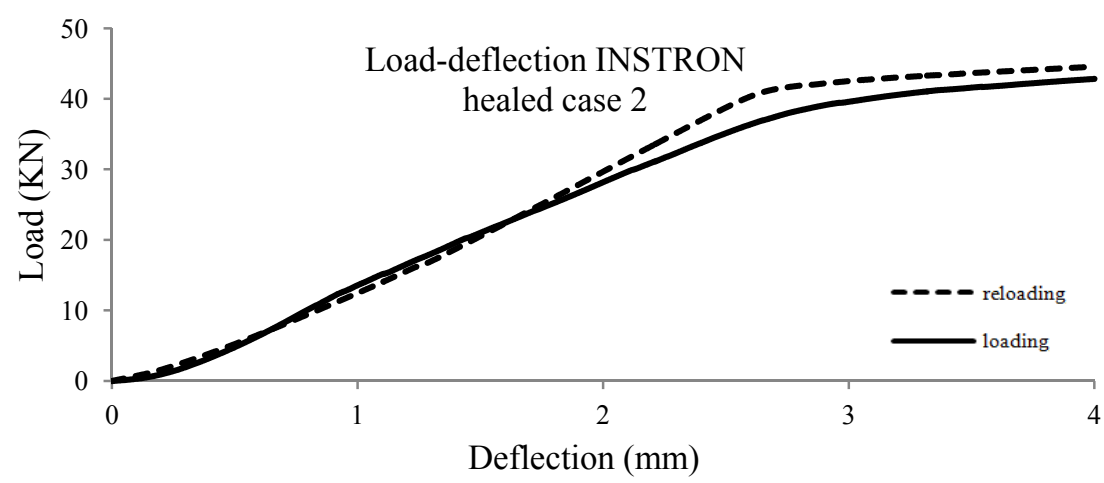

Figure 4: $\quad$ Load-deflection curve of the healed beam - case 2.

\subsection{Reference beams}

As is shown in the images below (Fig. 5), the bending behavior of the loading and reloading stage of the reference beam are quite similar. The reopening of the already existed cracks is verified by DIC analysis. Applying a straight line parallel and few millimeters above the bottom of the processed images, DIC software determine values of displacement and strain in (xx) direction (parallel to the steel reinforcement) for 200 points defined on this line. In the graph of crack opening related to the position of the 200 points (xx direction) that follows, one can see that the width of cracks is getting higher values at the case of reloading compared to the loading ones (Fig. 6).

Furthermore, there are no new cracks appearing at the reloading stage. Finally, as it is shown at the following strain profile of the beam (Fig. 7), the peak values of strain at the area of crack formation are higher in the case of reloading.

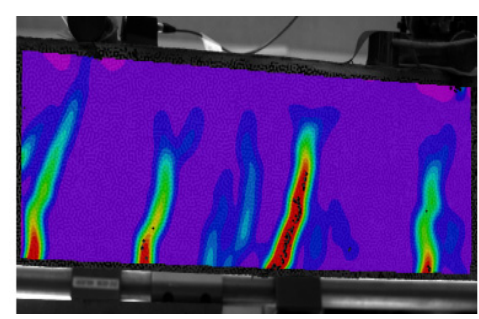

(A)

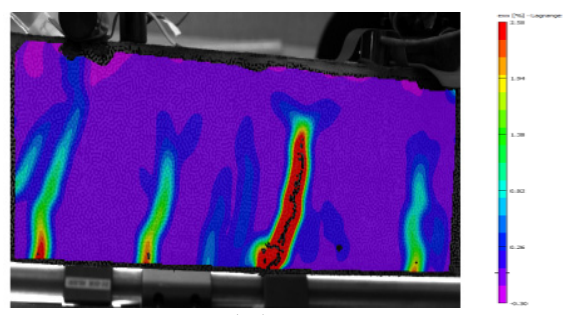

(B)

Figure 5: Crack pattern after loading (A) and reloading (B) of the reference beam. 


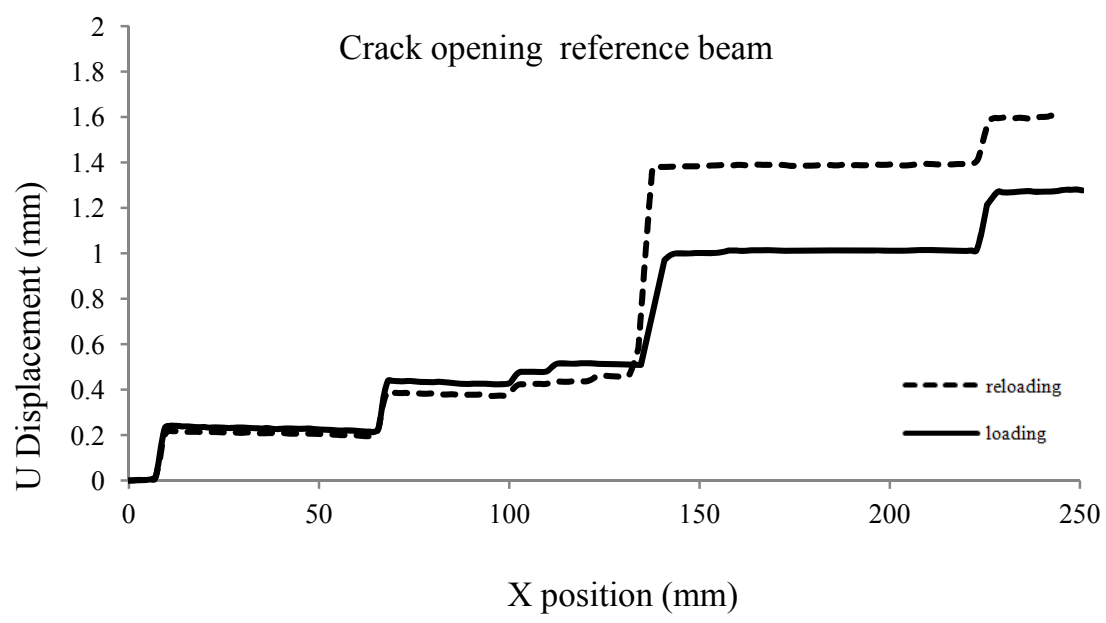

Figure 6: Crack opening at the bottom of the reference beam.

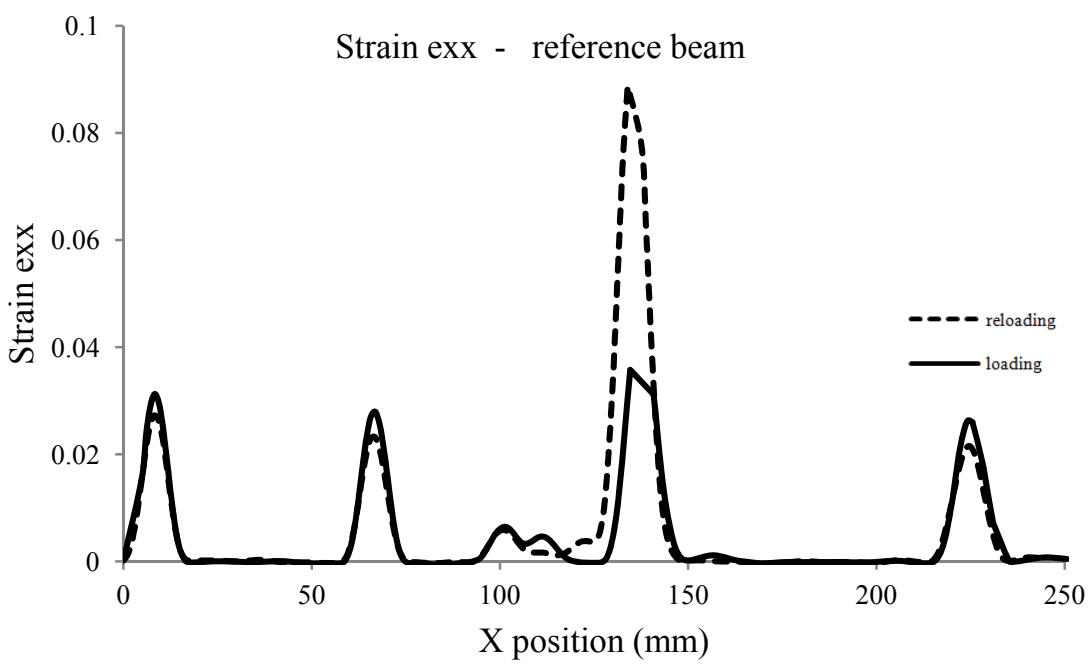

Figure 7: Strain distribution at the bottom of the reference beam.

\subsection{Healed beam: case 1}

In few of the beams a new crack seems to appear during reloading. This new crack formation is not visible by the INSTRON loading curve, but can be easily visualized at the following crack pattern (Fig. 8) of the loading and reloading stage of testing (healing system: short ceramic tube encapsulation protected by cement paste bar). Moreover, as it is noticed, there is a crack that is not formatted 
anymore at the stage of reloading. On the other hand, capsule breakage is filling this crack making the cracking area more resistant than before. Due to this reason, another weak point next to the initial crack is generating a new crack.

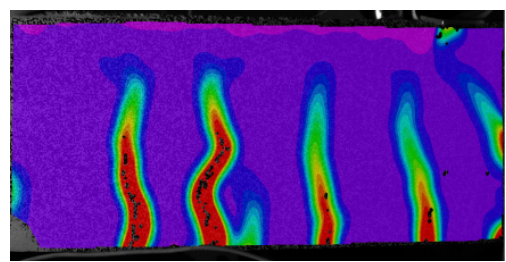

(A)

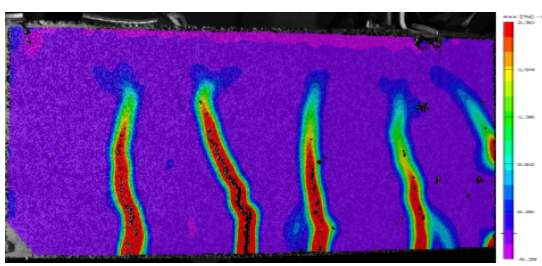

(B)

Figure 8: Crack pattern after loading (A) - reloading (B) of the healed beam - case 1 .

Quantitatively, the new crack opening is shown at the graph of displacement for 200 points of the line fixed at the bottom of the speckle pattern on the DIC analysis. The position in which the crack is formed at the reloading stage is changed obviously. Similarly, at the strain- position graph there is a peak of strain appearing on a different area of the beam compared to the initial loading stage (Figs. 9 and 10). Finally, the total opening of the cracks remains the same (since all the other cracks seem not to be healed by capsules breaking).

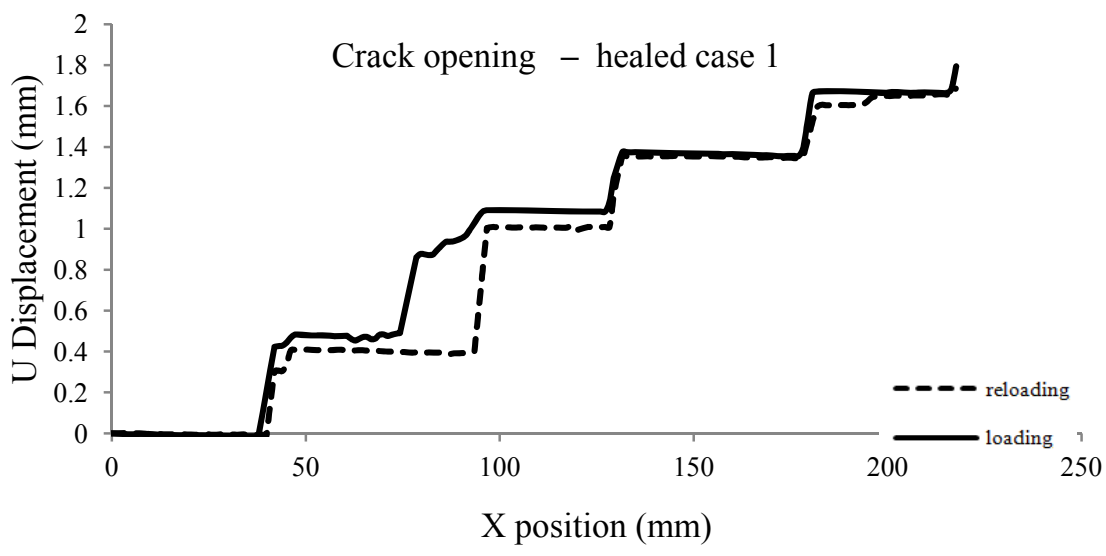

Figure 9: Crack opening at the bottom of the healed-case 2 beam. 


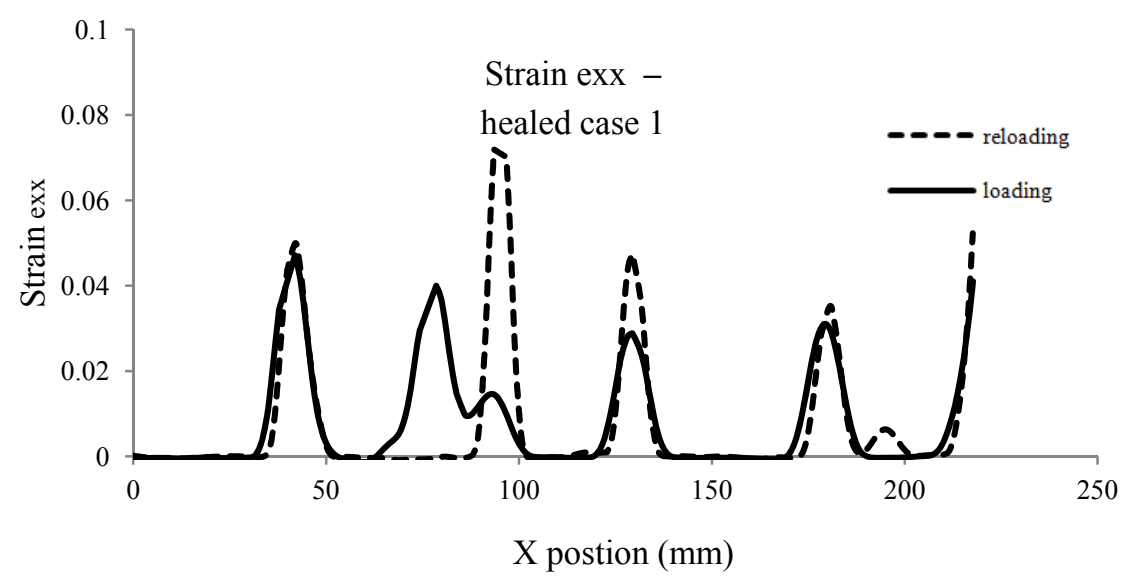

Figure 10: $\quad$ Strain distribution at the bottom of the healed beam - case 1 .

\subsection{Healed beam: case 2}

At most of the beams from different series (as mentioned and defined above according to the geometry and the type of capsule/ healing system) during reloading the already existed cracks reopen, but the width at the end of reloading got values lower than the width at the end of loading (Fig. 11). Apparently, cracks are filled by healing agent after capsule breakage but the already damaged cracking area is not strong enough to afford reloading and generates cracking again. The change in stiffness of this representative beam is obvious at the curve of loading-displacement derived from the INSTRON machine (healing system: short glass tube encapsulation protected by cement paste bar). The formation of less wide cracks was noticed during the DIC analysis taking into account the following crack patterns and graphs (Figs. 11-13).

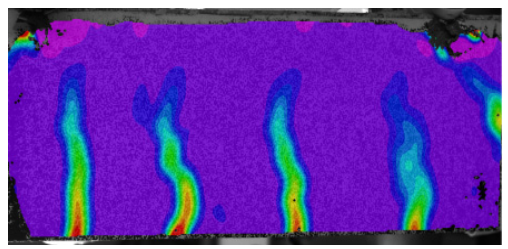

(A)

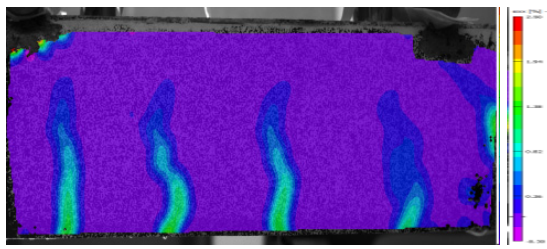

(B)

Figure 11: Crack pattern after loading and reloading of the healed beam case 2 . 


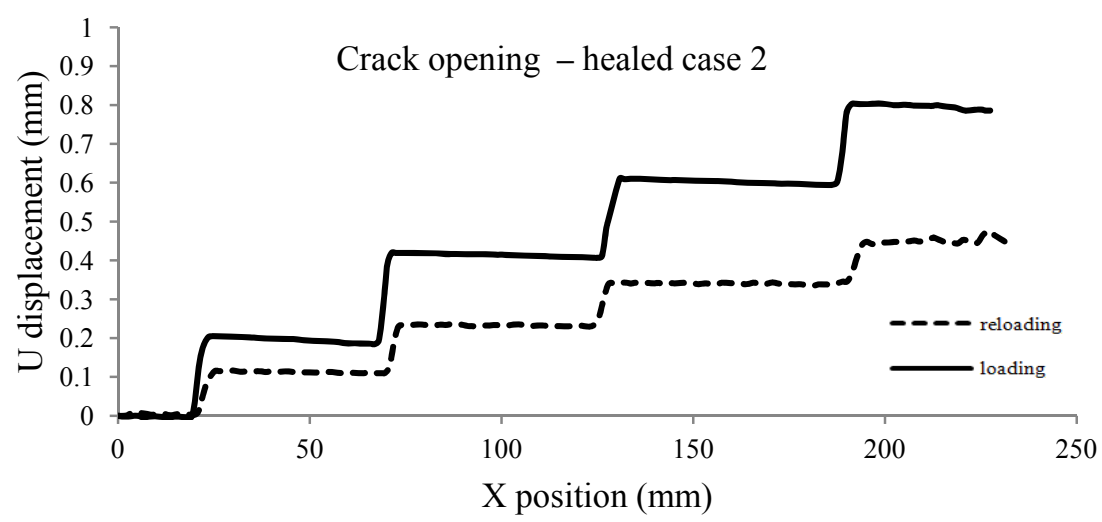

Figure 12: Crack opening at the bottom of the healed - case 2 beam.

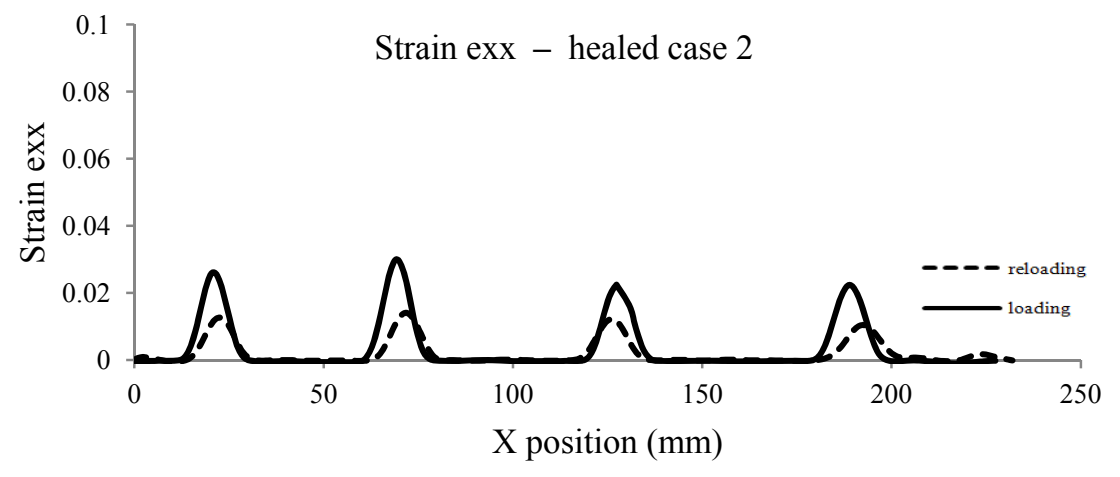

Figure 13: Strain distribution at the bottom of the healed - case 2 beam.

In the graph of crack opening it is shown that the sum of the crack width is taking values smaller at the reloading stage compared to the initial loading, but cracks seem to appear at the same defected area as before reloading (Fig. 12). Summarized and presented at the following table, the cracking width is getting the half of the loading value during reloading for all the cracks of this certain beam.

Table 3: Crack opening of the healed - case 2 beam.

\begin{tabular}{|l|c|c|}
\hline & $\begin{array}{c}\text { crack opening } \\
(\mu \mathrm{m}) \text {-loading }\end{array}$ & $\begin{array}{c}\text { crack opening } \\
(\mu \mathrm{m}) \text {-reloading }\end{array}$ \\
\hline 1st crack & 205 & 101 \\
\hline 2nd crack & 206,05 & 120,05 \\
\hline 3rd crack & 203,34 & 105 \\
\hline 4th crack & 200,63 & 109,48 \\
\hline
\end{tabular}


Further, strain peaks at the graph of strain-position (200 points of the bottom line) are getting lower values at the reloading stage (Fig. 13).Strain concentration phenomena around the healed cracks is further investigated in the near future.

\section{Conclusions: future work - fracture mechanics}

Full field DIC-images were used to investigate in full detail the cracking behavior of concrete with self-healing capabilities. It was found that, further conclusions cannot be drawn due to the limited number of beams tested in which healing phenomena appear.

In the near future, focusing at the phenomena around the crack tip will be combined with the fracture mechanics theories. The main aim of this study will be to prove the regain of mechanical properties due to healing by monitoring cracking phenomena.

Moreover, analysis of the promising results of the healing system applied can be used to obtain an experimental set-up that can successfully characterize mechanically the healing procedure.

\section{Acknowledgement}

The authors wish to acknowledge the IWT for financial support under the SIMEngineered Self-Healing Materials project.

\section{References}

[1] Van Tittelboom, K., De Belie, N., Self-healing concrete by the internal release of adhesive from hollow glass fibres embedded in the matrix, 2nd International conference on Self-Healing Materials, USA, 2009.

[2] Van Tittelboom, K., De Belie, N., Van Loo, D., Jacobs, P., Self-healing efficiency of cementitious materials containing tubular capsules filled with healing agent. Cement and Concrete Composites, 2011.

[3] Sutton, A, Orteu, J., Schreier, W., Image Correlation for Shape, Motion and Deformation Measurements, USA, 2009. 